\title{
Mitigating damage precursors to improve the laser resistance of ion beam sputtered antireflective coatings
}

Thimotheus Alig, Nils Bartels, Istvan Balasa, Tammo Böntgen, Detlev Ristau, et al.

Thimotheus Alig, Nils Bartels, Istvan Balasa, Tammo Böntgen, Detlev Ristau, Lars Jensen, "Mitigating damage precursors to improve the laser resistance of ion beam sputtered antireflective coatings," Proc. SPIE 11852, International Conference on Space Optics - ICSO 2020, $118521 D$ (11 June 2021); doi: $10.1117 / 12.2599237$

SPIE Event: International Conference on Space Optics - ICSO 2021, 2021, Online Only 


\section{International Conference on Space Optics-ICSO 2020}

Virtual Conference

30 March-2 April 2021

Edited by Bruno Cugny, Zoran Sodnik, and Nikos Karafolas
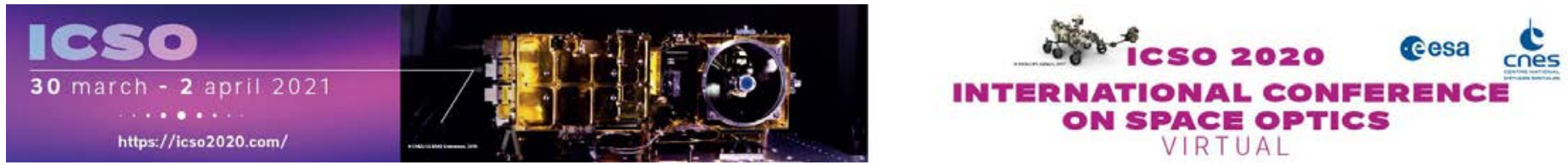

\section{Mitigating damage precursors to improve the laser resistance of ion beam sputtered antireflective coatings}

\section{Cesa issopocesalings lecnes}




\title{
Mitigating damage precursors to improve the laser resistance of ion beam sputtered antireflective coatings
}

\author{
Thimotheus Alig*a, Nils Bartels ${ }^{\mathrm{b}}$, Istvan Balasa ${ }^{\mathrm{a}}$, Tammo Böntgen ${ }^{\mathrm{a}}$, Detlev Ristau ${ }^{\mathrm{c}}$, Lars Jensen ${ }^{\mathrm{a}}$ \\ ${ }^{a}$ Laser Zentrum Hannover e.V., Hollerithallee 8, 30419 Hannover, Germany; ${ }^{b}$ Institute of Technical \\ Physics, German Aerospace Center (DLR), Pfaffenwaldring 38-40, 70569 Stuttgart, Germany; \\ 'Institute of Quantum Optics, Leibniz University Hannover, Welfengarten 1, 30167 Hannover, \\ Germany
}

\begin{abstract}
We manufactured and tested ion beam sputtered antireflection coatings for $355 \mathrm{~nm}$ using several defect mitigation strategies to improve the damage resistance of laser optics for LIDAR satellite missions. The first approach to avoid the damage precursors in the film is based on a secondary ion source. The goal is to remove the particles that settle on the surface of the growing film. A second strategy is to avoid the damage precursors in the ultraviolet wavelength range by laser conditioning of the thin films during the coating process. A laser beam at the application wavelength scans the deposited layer on the sample during deposition still in vacuum. Optical coatings produced with both technologies show a significantly increased damage threshold when tested in ramped raster scan tests.
\end{abstract}

Keywords: Laser-induced damage, antireflection coatings, ultraviolet spectral range, ion beam sputtering, aluminum oxide, silicon dioxide, damage precursors, ramped raster scan tests

\section{INTRODUCTION}

Satellite missions to study the atmosphere are becoming increasingly important due to the climate change. The Aeolus satellite for example provides much needed information to improve weather forecasting as part of ESA's Earth Explorer missions. One key part of the payload for these LIDAR satellite missions is the high power ultraviolet laser system. A major risk for the success of the missions is the damage resistance of the ultraviolet coatings within the laser system.

Damage precursors located in the dielectric coating are heated by the intense laser beam and causing a local plasma with temperatures of several 1000 Kelvin ${ }^{1,2,3}$. The mechanical stress inside the coating finally leads to damage sites by bursting off the overlaying material. The damage precursors can originate from grown lattice defects or particles in the deposition process. Additional precursors can be generated by the polishing process affecting the interface between substrate and layer system.

Damage growth is to be avoided in order to increase the reliability of the LIDAR systems against the intense laser radiation. The improved optics will also increase the sensitivity and accuracy by handling higher pulse energies of the transmitter in future. Within the scope of this work ion beam sputtered antireflection coating for $355 \mathrm{~nm}$ were manufactured and tested using two defect mitigation strategies.

*t.alig@1zh.de; phone +49 511 2788-0; fax +49 511 2788-100; lzh.de 


\section{METHODOLOGY}

The thin films were produced by ion beam sputtering motivated by the harsh conditions of a space mission. The samples with a residual reflection of $0.2 \%$ at $355 \mathrm{~nm}$ are designed with four layers of aluminum oxide and silicon dioxide. An ion beam source with neutralizer is used to sputter the coating material in reactive oxygen atmosphere. Since the coatings are antireflective, the laser radiation reaches all damage precursors down to the substrate surface. For this reason, an ultraviolet fused silica substrate with super polished surfaces was used. The mitigation schemes will be used to remove the damage precursors of the antireflection coatings during the manufacturing process.

\subsection{Ion based mitigation scheme}

The first approach to avoid the damage precursors in the film is based on a secondary ion source. The goal is to remove the particles that settle on the surface of the growing film. This was achieved by integrating a radio frequency pumped ion source into the coating system pointing towards the substrates (see Fig. 1). The secondary source extracts argon atoms with adjustable ion energy and beam current using a three-grid system made of titanium.

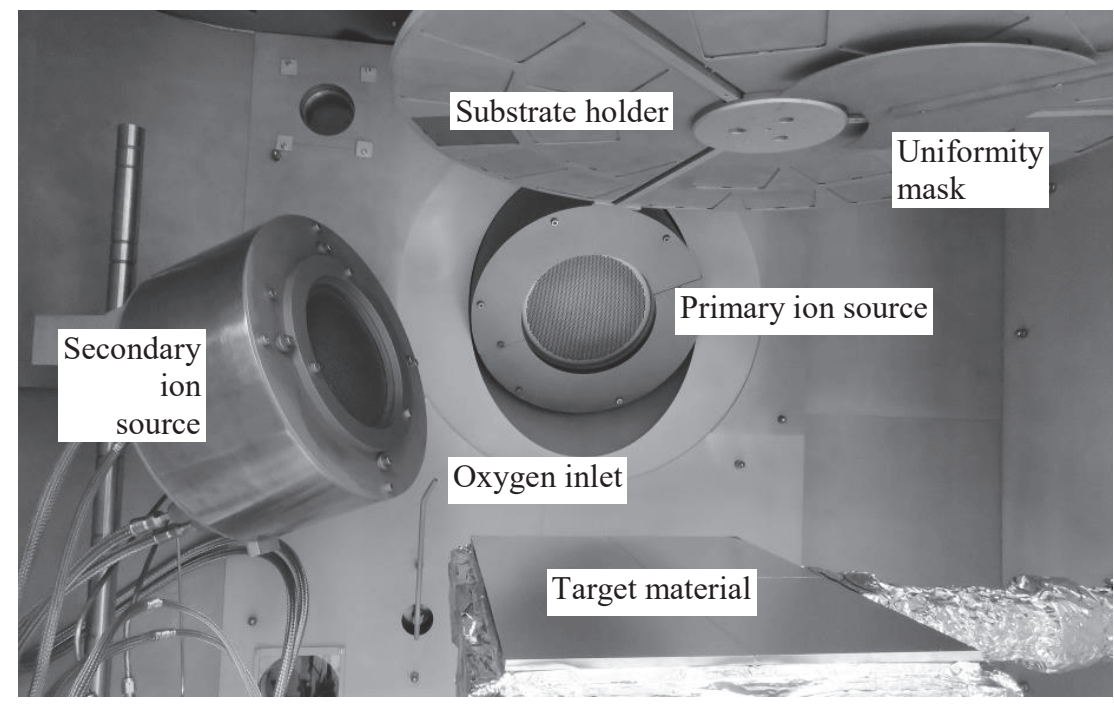

Figure 1. Setup of the ion beam sputtering system with the flexible mounting of the secondary ion source for the ion based mitigation scheme. The secondary ion bombardment in this configuration is applied at an incidence angle of $70^{\circ}$.

The ion based particle mitigation can be used on the super polished surfaces of the fused silica substrates. The second option includes ion bombardment during the deposition process. This resputtering process may also be used to reduce stoichiometric defects in the growing coating material.

In addition to the ion current and the ion energy, the angle of incidence can be adjusted using a flexible mounting system. It is possible to apply the ion bombardment with an ion energy of a few $100 \mathrm{eV}$, a beam current of a few $100 \mathrm{~mA}$, and an angle of incidence between 30 and $70^{\circ}$. The lateral momentum of the impinging particles is expected to distinguish the mitigation scheme from the dual ion beam sputtering process. 


\subsection{Laser based mitigation scheme}

The second strategy to avoid the damage precursors in the ultraviolet wavelength range is a laser conditioning of the thin films. This interaction can remove the precursors before they are imbedded within the film matrix. Resulting irregularities will be compensated by the coating material of the remaining layers.

The laser system provides pulses at the application wavelength and a duration of $8.3 \mathrm{~ns}$. The laser radiation with a power density of up to $14 \mathrm{~J} / \mathrm{cm}^{2}$ reaches the optical component through a window in the bottom of the deposition system. A galvanometer scanner outside of the chamber scans the sample line-by-line within a radius of $18 \mathrm{~mm}$ (see Fig. 2).

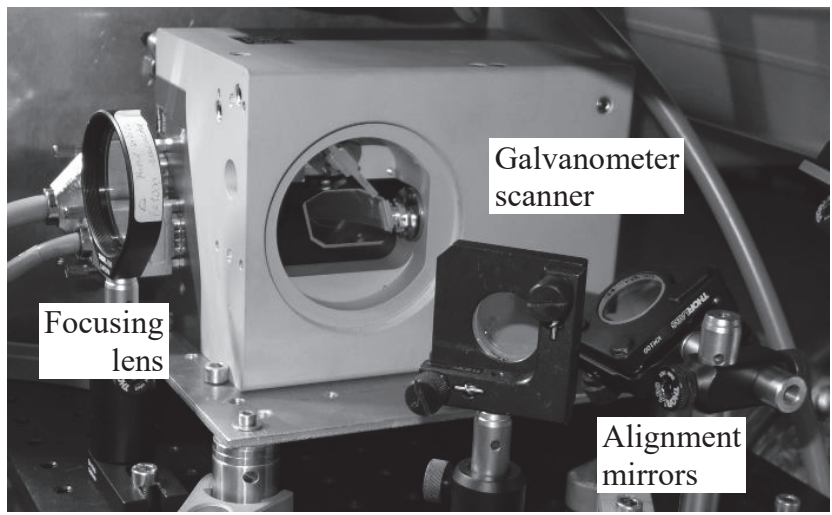

Figure 2. Setup of the laser based mitigation scheme with the galvanometer scanner under the ion beam sputtering system.

The laser irradiation at the application wavelength can address damage precursors on the substrate surface similar to the ion based mitigation approach. The second step of the laser based particle mitigation involves an additional irradiation after each deposited layer. The impact on the damage resistance is investigated in dependence of the energy density as well as the number of pulses.

\section{RESULTS}

In the first part of this study the damage behavior of different process configurations are compared to optimize the particle mitigation schemes. In order to investigate in the laser induced damage behavior, raster scans of the samples with an energy density of $25 \mathrm{~J} / \mathrm{cm}^{2}$ were performed. Dark field microscopy of the antireflection coatings before and after the raster scan tests was used to detect the laser-induced damage sites.

The ion based particle mitigation was initially used on the super polished surfaces of the fused silica substrates. Employing an angle of incidence of the argon ions of $70^{\circ}$, a slightly reduced damage density compared to $45^{\circ}$ and $30^{\circ}$ was achieved. When applied during the deposition process the oblique ion bombardment resulted in a significant reduction of the laser-induced damage sites. The improvement in damage resistance increased with both the ion energy and the beam current of the secondary bombardment (see Ref. 4 for details).

The influence of the laser based mitigation scheme was investigated in dependence of the energy density as well as the number of pulses. In accordance with the ion based mitigation scheme, only minor improvements could be achieved by a substrate pretreatment. The application of the particle mitigation after each layer showed a significant improvement increasing with the energy density and the number of pulses.

Following the optimization, ramped raster scan tests were performed to compare the damage threshold of the improved optics against the reference samples defined by identical coatings produced in the same deposition plant without applying the ion source or laser system for particle mitigation. The raster scan tests were performed in steps of $5 \mathrm{~J} / \mathrm{cm}^{2}$ up to a maximum energy density of $30 \mathrm{~J} / \mathrm{cm}^{2}$ applying a number of 10 pulses. The raster scan tests with an area of $10 \times 3 \mathrm{~mm}^{2}$ were evaluated using dark field microscopy before and after the irradiation similar to the optimization procedure. 
Special areas of the reference system were analyzed to observe the development of damages for different energy densities (see Fig. 3). A coating defect was identified under the Nomarski microscope before performing the first raster scan test. This coating defect in the reference system expands after the raster scans with energy densities of 15 and $25 \mathrm{~J} / \mathrm{cm}^{2}$ up to an equivalent diameter of about $10 \mu \mathrm{m}$.

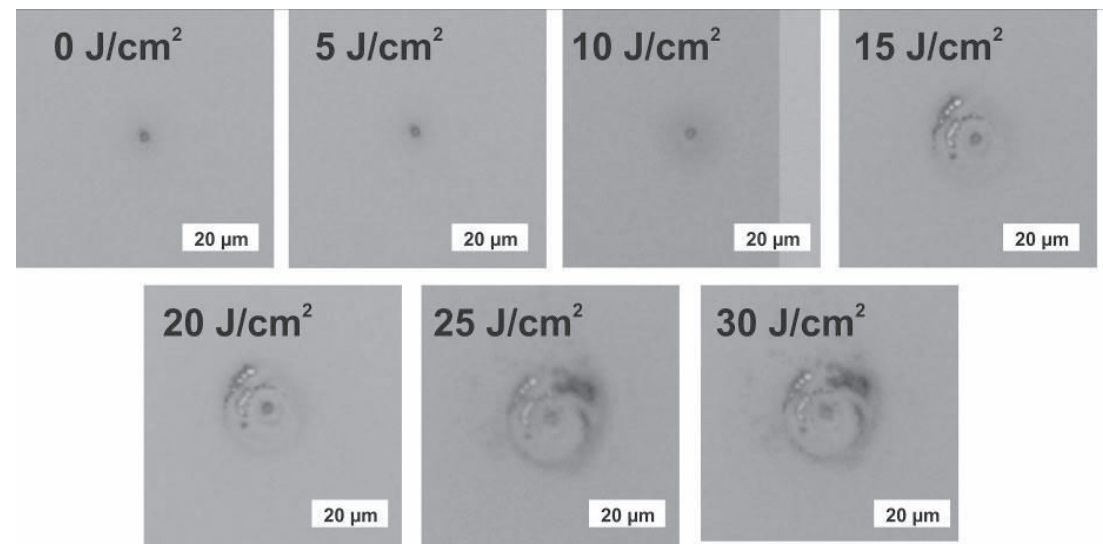

Figure 3. Development of a pre-irradiation coating defect on the standard optic under the Nomarski microscope within the ramped raster scan test.

The reference system was measured against the ion based mitigation scheme taking a beam current of $200 \mathrm{~mA}$ and an ion energy of $250 \mathrm{eV}$ (see Fig. 4). The samples of the laser based mitigation scheme were produced with an energy density of $10 \mathrm{~J} / \mathrm{cm}^{2}$ and a number of 20 pulses.

A computer based brightness evaluation was employed to analyze the dark field micrographs of the ramped raster scan tests for scattering objects. The special software masks areas around pre-irradiation coating defects to concentrate on laser-induced damage sites (see Ref. 5 for details). The results obtained with the masking algorithm were confirmed performing also an evaluation of all damages after the ramped raster scan tests. In order to distinguish between generated damage sites, a classification of the detected area was made based on the equivalent diameters. 


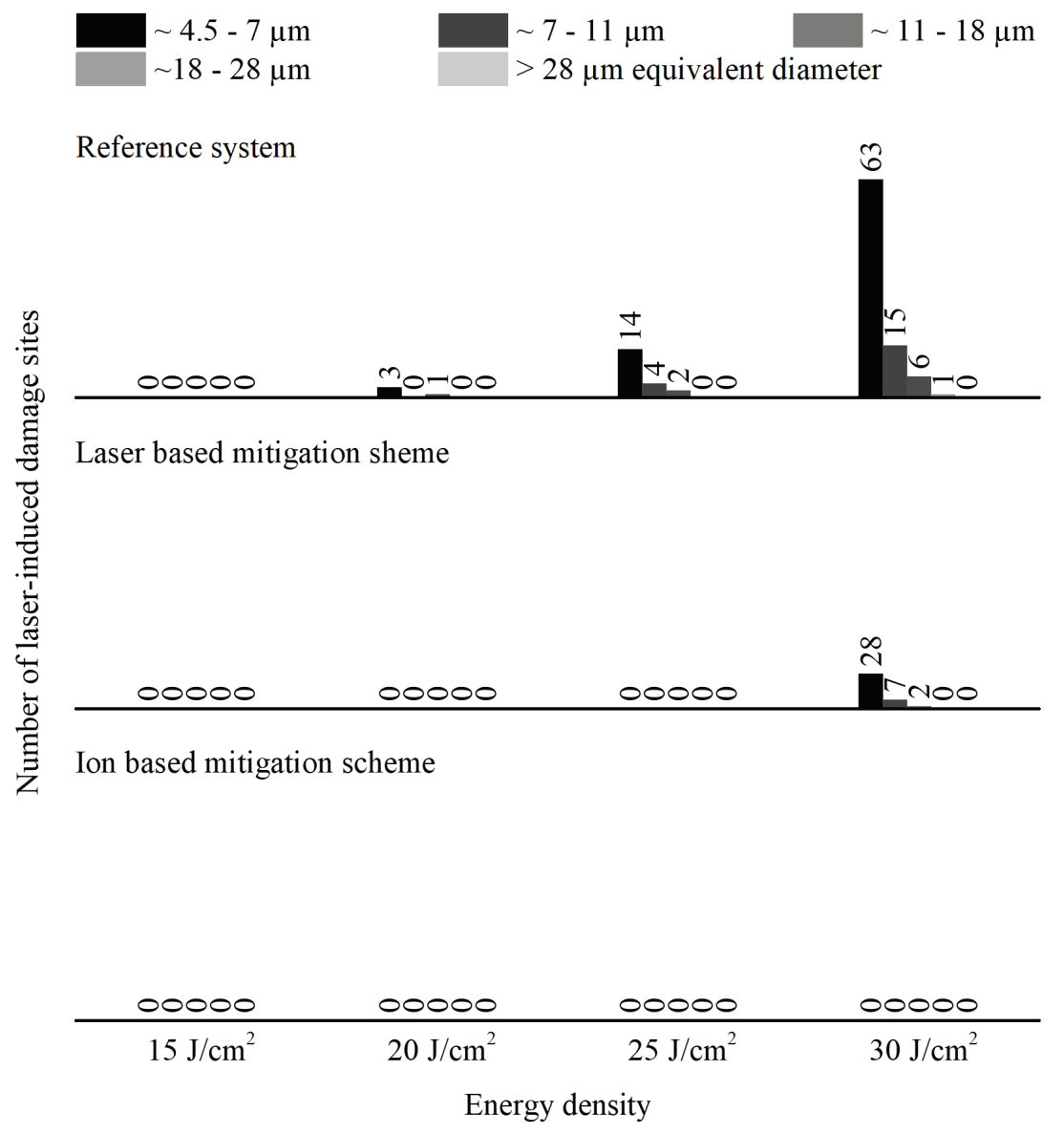

Figure 4. Number of laser-induced damage sites of standard optic, laser based and ion based mitigation scheme in the ramped raster scan test neglecting areas with initial coating defect.

Laser-induced damages appeared first in the reference coating after the raster scan test with an energy density of $20 \mathrm{~J} / \mathrm{cm}^{2}$. Considering the successive increase of the energy density the influence of laser conditioning on the antireflection coatings should be taken into account ${ }^{5}$. The antireflection coating produced with the laser based particle mitigation shows laser-induced damages at an energy density of $30 \mathrm{~J} / \mathrm{cm}^{2}$. In case of the ion etched thin films, the number of detected objects did not increase at all indicating a laser-induced damage threshold of above $30 \mathrm{~J} / \mathrm{cm}^{2}$.

\section{CONCLUSIONS}

Both mitigation schemes revealed that the optimization of the coating requires a removal of damage precursors within the coating layers. The elimination in the laser based mitigation scheme already took place before a significant amount of layer material was added and no detectable crater was formed. The damage precursors in the ion based mitigation scheme were removed by introducing the oblique angle of the secondary ion bombardment. The mitigation scheme increased the laser-induced damage threshold by at least $50 \%$ compared to the conventional deposition process. This data shows, that this technology can make a valuable contribution to applications with a demand for high reliability laser optics, e.g. for future lidar space missions. 


\section{ACKNOWLEDGMENTS}

This work has been carried out under the ESA contract AO 1-8683/16/NL/BJ "Particle mitigation in high power laser optics". The authors would like to thank Ana Baselga Mateo (ESA-ESTEC) and Clemens Heese (ESA-ESTEC) for support and scientific advice during the initiation and execution of this project.

\section{REFERENCES}

[1] L. G. DeShazer, B. E. Newnam, and K. M. Leung "Role of coating defects in laser induced damage to dielectric thin films", Appl. Phys. Lett. 23, 607 (1973).

[2] Papernov, S., and A. W. Schmid. "Correlations between embedded single gold nanoparticles in SiO 2 thin film and nanoscale crater formation induced by pulsed-laser radiation." Journal of Applied Physics 92.10 (2002): $5720-5728$.

[3] Negres, Raluca A., et al. "Growth behavior of laser-induced damage on fused silica optics under UV, ns laser irradiation." Optics Express 18.19 (2010): 19966-19976.

[4] T. Alig, N. Bartels, P. Allenspacher, I. Balasa, T. Böntgen, D. Ristau, and L. Jensen, "Prevention of laser damage precursors in spaceborne ultraviolet antireflection coatings", Optics Express ,2021 (submitted).

[5] N. Bartels, P. Allenspacher, T. Alig, I. Balasa, H. Schröder, G. Taube and W. Riede. "Laser-induced damage and contamination testing for the next generation of LIDAR space optics", ICSO, 2021

[6] Bartels, N., Allenspacher, P., \& Riede, W. (2018, November). Laser conditioning of UV anti-reflective optical coatings for applications in aerospace. In Laser-Induced Damage in Optical Materials 2018: 50th Anniversary Conference (Vol. 10805, p. 108051Q). International Society for Optics and Photonics. 